\title{
Pandemic and Panic: Government as the Supplier of Last Resort ${ }^{1}$
}

\author{
Yi Huang (Graduate Institute and CEPR) \\ Chen Lin (Faculty of Business and Economics, the University of Hong Kong) \\ Pengfei Wang (PHBS, Peking University and HKUST) \\ Zhiwei Xu (Shanghai Jiao Tong University)
}

\section{Introduction}

The Covid-19 pandemic has caused a global health and economic crisis. As of March 29,2020 , the number of confirmed cases has surpassed 650,000 , with over 30,000 deaths around the world so far. The pandemic has also sparked panic buying and hoarding of critical medical products by members of the public, as well as a significant surge in unnecessary hospital visits. How governments deal with these issues is critical to their overall response to the pandemic and ability to end the crisis.

When the coronavirus outbreak started in China, concerned citizens started panic buying medical products such as masks almost immediately. Hospitals were also quickly flooded by patients worried they had contracted the virus, which severely strained their resources and meant that many patients with serious conditions unrelated to the virus went untreated.

Economists in China engaged in a heated debate about whether and how the government should regulate the prices of affected medical products. There were generally two perspectives. Pro-market advocates argued that the panic buying reflected a significant and actual discrepancy between supply and demand, and the government should therefore not interfere by imposing price regulations. The surge in market prices was a strong signal for manufacturers to increase production. Others argued, though, that supply and demand was too simple a lens to apply to price dynamics during periods of turmoil when people are not being rational actors. The invisible hand, they said, had failed to help the market achieve efficient allocation. However, both arguments are based upon canonical demand-supply theory - perhaps we could think about the issue from a different angle.

In this essay, we argue that there is an analogy between panic buying of masks and historical bank runs. For many people, the pandemic has created the impression that

\footnotetext{
1 We would like to thank Bengt Holmström, Wenlan Qian, Yijiang Wang, Liyan Yang and especially Yi Wen for thoughtful discussions and suggestions. We also thank Melody Guo for research assistance.
} 
masks are a necessity. While the surge in demand and prices is indeed a spontaneous market reaction, the demand is largely driven by precautionary motives.

In this sense, the surging demand for medical products such as masks is much the same as a bank run. ${ }^{2}$ In this article, we will study the underlying mechanisms behind the panic buying of masks from the perspective of a classic bank run. Understanding the principle behind this phenomenon could be helpful for governments to address the root cause of the problem.

A bank run occurs when a large number of clients suddenly pull their money out of a bank. Banks usually do not keep 100 percent reserve funds. When faced with runs, even strong banks will face a liquidity crisis and fall into bankruptcy, which makes a bank run a self-fulfilling prophecy. During a mask shortage, the bank corresponds to a retailer and the bank reserve corresponds to its stock of masks.

Bank runs have caused economic crises repeatedly throughout history. In 1930, for instance, the US economy had begun to recover from the stock market crash one year earlier. Unfortunately, a series of bank runs pushed the US economy into the Great Depression. Bank runs have been recorded as early as the 17 th century, although a formal theory of bank runs in economics was not developed until 1983. Diamond and Dybvig, two American economists, used simple math to describe in detail the bank's function of liquidity creation. The logic behind the theory is very intuitive. In general, investors demand long-term funds to finance their projects while savers demand shortterm liquidity to meet their current needs, with banks pooling the funds of both parties to solve the liquidity mismatch problem. In normal times, banks hold a certain amount of reserves and the remaining funds are provided to investors in the form of long-term loans.

However, Diamond and Dybvig proved there is bank run equilibrium. The mechanism works as follows. The bank intrinsically cannot recall long-term loans to meet shortterm demand for liquidity because it does not maintain sufficient reserves. Once depositors become concerned about the risk of a bank run, they rush to withdraw their money before others to ensure the safety of their funds. This panicked behavior creates negative consequences and shared pain for individuals, leading to market failure.

Back to the panic buying of masks caused by the coronavirus pandemic. Like bank runs, the panic prompted many people to purchase and hoard masks before they sold out, which we will refer to as a "panic run." Due to limited daily production capacity, this behavior placed people with an urgent need for masks, such as medical workers, at even higher risk. This in turn slowed containment efforts, which again increased the frenzied

\footnotetext{
2 See Wang and $\mathrm{Xu}$ (2020) for more recent discussions about the mask run in China during the outbreak of
} coronavirus. 
public demand for masks. Even worse, sellers began to anticipate that prices would surge further and started hoarding masks in anticipation of greater profits, exacerbating the shortage. This spiral caused the market to fail to allocate masks efficiently. In the following section, we provide a simple model to regulate panic run behavior.

\section{A Simple Panic Run Model}

We consider a two-period simple model, $t=1,2$. The economy has one unit measure of population. There are two types of people: the low infection risk group (type L) with measure $\alpha \in(0,1)$ and the high infection risk group (type $\mathrm{H}$ ) with measure $1-\alpha$. We assume there is a medical product that can protect people from infection. In the model, we use masks as a specific example. The main analysis also applies to other protective products or medical services relating to the coronavirus.

In period 1 , the producer can supply $y_{1} \leq m<1$ units of masks at a marginal cost of $\phi>0$. In period 2 , the producer can supply $m-y_{1}$ units of masks at a marginal cost of $\phi>0$. We assume that the type $\mathrm{H}$ agents will be infected with the virus in the absence of the protection of a mask. However, in period 1 there is zero risk of the type $\mathrm{L}$ agents being infected.

We assume that the momentum disutility of infection is $-V$, hence the utility gain derived from wearing a mask is $V$ for type $\mathrm{H}$ agents, and zero for type $\mathrm{L}$ agents. We assume that the type L agents will become the high-risk type in period 2 with probability $\theta \in(0,1)$ due to the spread of the virus, and wearing a mask can prevent them from being infected. So the expected utility of wearing a mask for type $\mathrm{L}$ agents is $\theta \mathrm{V}$. We assume that $V$ is sufficiently large such that

$$
\theta V>\phi
$$

In period 2 , the number of infected agents is $1-\alpha+\alpha \theta$. We further assume that the total stock of masks $m$ satisfies

$$
1-\alpha+\alpha \theta<m<1 \text {. }
$$

The last condition implies the stock of masks can cover all of the agents with high exposure risks in the first two periods, but it cannot meet the demand if every agent buys masks in period 1 . We assume in the latter scenario that masks are sold on a firstcome first-serve basis. In the case of medical services, $m$ may correspond to the number of frontline healthcare workers.

\subsection{A Panic Run under Exogenous Price}

We first consider a case of exogenous price where the price of a mask in two periods is equal to the marginal cost, i.e. $P_{1}=P_{2}=\phi$. As in the Diamond-Dibvig bank run model, there exist two equilibria: the normal equilibrium, where no agent engages in 
surge buying of masks; and the panic run equilibrium, where the panic buying of masks occurs in period 1.

Normal Equilibrium Only type H (high-risk) agents buy masks in period 1, and type L (low-risk) agents just wait and buy masks in the second period if they become the high-risk type. During the equilibrium, the net utilities (compared to the situation without the protection of masks) for both types of agents satisfy

$$
U_{H}=2 V-\phi \text { and } U_{L}=(V-\phi) \theta \text {. }
$$

The total demand for masks is then given by $1-\alpha+\alpha \theta$, which is lower than the total supply $m$. Therefore, a run does not occur. Notice that each agent's decision is optimal in the sense that no one has the incentive to deviate. Given others' strategies, individual type $L$ agents obtain a net utility of $-\phi+V \theta$ if this deviates, which is lower than $U_{L}$. For each type $\mathrm{H}$ agent, it is obvious that the deviation drives a net utility of zero, which is lower than $U_{H}$.

Panic Run Equilibrium In this equilibrium, if both types of agents rush to buy masks in period 1 , then the total demand is 1 . However, each of them can get the masks only with a probability of $m$ due to the limited supply. Therefore, the expected net utilities for both types of agents are given by

$$
\widehat{U}_{H}=(-\phi+2 V) m \text { and } \widehat{U}_{L}=(\theta V-\phi) m .
$$

Again, in this panic run equilibrium, no one has the incentive to deviate since they will obtain a zero net utility if they deviate and wait until the next period. Therefore, these two equilibria are self-fulfilling.

\subsection{Pricing Mechanism}

One natural question is whether the market mechanism, i.e. raising the price, can solve the problem of the shortage caused by the run. To answer this question, we further endogenize the price of masks. We will show that it is more likely to cause opposition. The market pricing mechanism may worsen the severity of the mask shortage and cause the worst-case scenario.

We assume that in period 1 the price is fixed at marginal cost, i.e. $P_{1}=\phi$, and the market condition endogenously determines the price in period 2. For the supply side, we now allow the sellers to decide how many masks to sell in period 1 . If the sellers believe $P_{2}>P_{1}=\phi$, they may have a strong incentive to hoard the masks in period 1 and sell them afterward.

Let $s$ denote the fraction of high-risk agents who fail to buy the masks in the first period. We assume that the probability of type L agents becoming type $\mathrm{H}, \theta$, is increasing in $s$, i.e. $\theta^{\prime}(s)>0$. This assumption characterizes the positive externality 
of wearing the masks during the pandemic. In the second period, $\theta(s) \alpha+(1-$ $\alpha)(1-s)$ fraction of the total population is under high exposure risks, who demand the protection of masks. Thus, the total demand for masks in period 2 is $\theta(s) \alpha+(1-$ $\alpha)(1-s)$. A lower fraction of type $\mathrm{H}$ agents obtaining masks in period 1 ( $s$ is high) induces a higher demand for masks in period 2. We further assume that the stock of masks $m$ satisfies

$$
\theta(0) \alpha+1-\alpha<m<\theta(1) \alpha+1-\alpha .
$$

The above condition implies that the maximum production of masks can cover the demand in a normal situation where every type $\mathrm{H}$ agent can obtain masks in period 1 (i.e. $s=0$ ), but it cannot meet the demand under a panic situation where no type $\mathrm{H}$ agents get masks (i.e. $s=1$ ). Besides, analogous to the case of exogenous price, we assume $\theta(0) V>\phi$. Again, there exist two equilibria.

Normal Equilibrium In this equilibrium, no panic run occurs. The prices in two periods are equal to the marginal cost, $P_{1}=P_{2}=\phi$. The sellers behave competitively and receive zero profit. The net utilities for both types of agents are $U_{H}=-\phi+2 \mathrm{~V}$ and $U_{L}=(V-\phi) \theta(0)$, respectively. Analogous to the previous analysis, in this equilibrium each agent has no incentive to deviate given others' strategies. Sellers also have no incentive to deviate. This is because the total demand in period 1 and period 2 is $\theta(0) \alpha+1-\alpha$, which is less than the total supply of masks $m$, implying a buy-side market. Therefore, the price $P_{2}$ must equate to the marginal cost $\phi$. The zero-profit condition deters the sellers' hoarding behaviors for masks in period 1, which confirms the equilibrium conditions on the demand side.

Panic Run and Hoarding In this disequilibrium, similar to the case of exogenous price, the panic run occurs. However, the situation becomes even worse when the market conditions endogenously determine the price. Since we assume that the price is fixed in period $1\left(P_{1}=\phi\right)$, the sellers now have a strong incentive to hoard all of the masks and wait until the second period to sell them. This hoarding behavior is optimal for the sellers since, in period 2, they can sell at a higher price. To see this, remember that if the sellers hoard the masks, no agent, including the high-risk group, can get masks in period 1, i.e. $s=1$. Therefore, in period 2 the total demand for masks is $\theta(1) \alpha+1-\alpha$, which exceeds the total supply $m$ (see Eq. 5). The market for masks is now on the seller's side, where the price is set to the marginal value of the buyer, $P_{2}=V$. This equilibrium is the worst scenario with the lowest level of social welfare, as none of the high-risk agents can obtain the masks in the first period, and more people are exposed to the virus.

\section{Policy Implications and China's Experiences}

The question now becomes: How could we alleviate the panic buying of scarce medical 
resources during a pandemic? The bank run theory can inspire a potential solution. After the invention of the Diamond-Dybvig model, economists wrote thousands of papers on how to end bank runs. Two measures are mentioned most often. The first is to stop cash withdrawals to prevent a bank run from the demand side. The other is to establish a deposit insurance system (DIS) to consolidate the safety of deposits from the supply side. The DIS requires a central bank that can provide unlimited liquidity to support it.

Two similar measures could help solve the problem of panic runs. The first is to directly curb demand. In the case of a mask shortage, this could be achieved by imposing tighter restrictions on the buying of masks in those areas less severely affected by a virus, or creating a lottery to limit mask purchases. For example, if the government mandated that exactly $1-\alpha$ unit of masks should be sold in the first period, this would eliminate the panic run equilibrium in the first fixed-price equilibrium. Knowing there will be $m-(1-\alpha)>\alpha \theta$ masks left in the second period, type $\mathrm{L}$ agents would have no incentive to buy masks in the first place. However, this is feasible only if the government knows the true fraction of both types. As discussed above, if the seller can increase their price in the second period, government restrictions on purchases in the first period would not be useful.

In the early stage of the coronavirus outbreak in China, some of the above measures were implemented by local governments. Similarly, in the case of medical services, the policy could be to require infected patients with mild symptoms to self-isolate. However, it should be noted that although the above measure could curb the panic run, it does not substantially enhance allocation efficiency or optimize social welfare. Even worse, there would be a potential risk of increasing transmission of the virus.

The second potential measure is for the government to establish its own production base. For medical products such as masks, it is not profitable for other enterprises to switch to mask production for a short period due to slow regulatory approval processes and the fact that the surge in demand is temporary. Thus, the market has limited ability to expand the supply in the short term. Therefore, as a last resort, the government should quickly begin its own production while still guaranteeing product quality. These production facilities should be part of the national public health system, which plays a similar role as the deposit insurance system as a supplier of public goods. After all, during a pandemic, a critical medical product such as a mask becomes a public good because it creates a positive externality by curbing the spread of the virus.

When the coronavirus outbreak began in Wuhan, the whole city experienced an acute shortage of medical products and services such as N95 surgical masks and hospital beds. The Chinese government then issued a series of virus-containment edicts that generally 
followed the logic of the second type of measures discussed above. ${ }^{3}$ The central government also created a stimulus package, including fiscal and monetary instruments for the expansion of production capacity for anti-virus-related goods and services. According to official statistics, the production capacity of masks in China increased from 20 million per day to 116 million per day within a month. This rapid expansion in the supply largely mitigated the public panic over masks. To solve the acute shortage of hospital beds amid the outbreak, China also started building temporary hospitals almost immediately. When Wuhan went on lockdown, two temporary hospitals, the Huoshenshan and Leishenshan facilities, with a combined total of 2,600 beds, were completed and started to operate within two weeks. Also, the Wuhan municipal government built 14 temporary medical stations to treat patients with mild symptoms. Many other cities also built similar hospitals as a precautionary measure. Other provinces around China sent more than 40,000 doctors and nurses to Hubei province to support frontline healthcare. These measures largely mitigated public panic over the availability of medical services, and effectively stemmed the spread of the virus in that country.

To prepare for the next pandemic, governments should create strategic inventories of essential medical supplies and equipment. These strategic inventories can increase the overall systematic capacity to treat patients. At the same time, as this essay suggests, they are effective measures to reduce panic runs, which will allow scare medical resources to be allocated to those who need them most.

\section{References}

1. Diamond, D.W. and Dybvig, P.H., 1983. Bank runs, deposit insurance, and liquidity. Journal of Political Economy, 91(3), pp.401-419.

2. Huang, Yi, Lin, Chen, Wang, Pengfei and Xu, Zhiwei, 2020. Saving China from the coronavirus and economic meltdown: Experiences and lessons, 2020, in VOXEU e-book Mitigating the COVID Economic Crisis: Act Fact and Do Whatever It Takes, link: https://voxeu.org/article/saving-china-coronavirus-and-economic-meltdown-experiencesand-lessons

3. Wang, Pengfei, and Xu, Zhiwei, February 02, 2020. Mask Panic as Bank Run (in Chinese), Yicai, link: https://www.yicai.com/news/100485707.html

\footnotetext{
${ }^{3}$ See Huang, Lin, Wang and Xu (2020) for more recent discussions about the anti-virus policies conducted by the Chinese government.
} 